\title{
Android Based Body Temperature, Heart Beat and Drouse Sensor Monitoring Using and SMS Based Telemedicine System
}

\author{
Rajeshwari S, Arpitha P, Meghana P,Sushmitha C \\ Asst. Professor, Dept of Information Science and Engineering, \\ $8^{\text {th }}$ sem student Dept of Information Science and Engineering, \\ RajRajeswari College of Engineering, VTU, Bangalore, India
}

\begin{abstract}
The primary concentration of this paper is to execute a model for the constant patient checking framework. This framework is utilized to quantify crucial parameters, for example, body temperature, pulse, ECG, glucose and tiredness uses the remote systems. The primary objective of this framework is to build community oriented and cost productive gadget which is moderate for ordinary citizens, the patients who are in the ICU, home care. The application has been created to store the information that can additionally downloaded by the doctor workstation for further examination. The trouble in today's life is transport office in the shrewd cities. In this paper we propose a patient checking framework which can be worked remotely. In this paper we talk about and building up a minimal effort mechanical assembly which utilizes GSM innovation for observing well- being state of the patient.
\end{abstract}

Keywords: GSM, Heart Rate, ECG, ICU, Home care.

\section{INTRODUCTION}

Nonstop estimation of patient parameters, for example, temperature, respiratory rate, circulatory strain and numerous others have turned out to be normal component of the instance of fundamentally is patients. Whenever precise and quick basic leadership is urgent for viable patient care, electronic screens are much of the time need to gather and show physiological information, such information are gathered using nonintrusive sensors from patients in a clinics therapeutic surgical units, work and conveyance suites, nursing home or in patients claim house to recognize an unforeseen life threaten occasions in patients basically sick.

Understanding checking framework can be characterized as "rehashed or nonstop perceptions or estimations of the patient, his or her physiological capacity and the capacity of life bolster hardware with the end goal of directing administration choices. A patient screen may not just ready guardians to possibly undermining occasions; many additionally give physiologic info information used to control specifically associated life bolster gadgets. The correct usage of such framework can give convenient notices to the restorative staffs and specialists and their administrations can be initiated if there should be an occurrence of therapeutic crises.

The whole information or the data got is put away in cloud. It keeps up the patient's life time restorative points of interest in a solitary archive and can be gotten to at whatever point and wherever required.
We talk about the utilization of PCs to help parental figures in the gathering, providers in the accumulation, show, stockpiling and basic leadership including the translation of clinical information making restorative proposal and disturbing and cautioning utilizing IOT gadgets The conclusion that we are exhibiting in this paper it depict how the work make a stage for a definitive objective of customized prescient observing.

\section{LITERATURE SURVEY}

1.The framework used to screen the essential parameters of human subject comprises of gadget which can be worn into a wrist and finger by an at hazard individual. Three sensor can be utilized by the individual which can be remotely observed in his own particular house from the remote place. As an effect the sensor distinguishes the restorative parameters and sends an alert to individual collector.

2.Later on the innovations composed and built up a framework more dependable, effective and screen the patient from the remote place, which is fit for sending parameters of patients in a constant. It likewise empower the information to screen patients parameters.

3.A biomedical signs checking framework with numerous physiological signs estimation ability progressively is planned and executed. This framework plays out a nearby crucial sign information examination utilizing phone over remote sensor arrange (WSN) innovation. Mobile phone based individual medicinal recorder winds 
up noticeably prominent to encourage discretion of incessant illnesses and watch patients' condition constantly.

The danger of disregarding observing patients' physiological information by specialist is worried since extensive amounts of information are handled and dissected day by day. A smart choice emotionally supportive network is created to upgrade medicinal services suppliers' audit of observing information.

4.In this paper we display a constant, consistent essential signs observing framework in view of a Multi-operator design. Ongoing remote observing of the patient's essential signs and producing alarms if there should arise an occurrence of crisis circumstances is of awesome significance in social insurance. A few modest sensors appended to the patient's body can gather physiological estimations of key signs, and transmit them to a remote server, from where it can be counseled by a doctor. Utilizing Wireless Body Area Networks (WBANs) innovation patients can move unreservedly and perform day by day assignments while being checked. In such frameworks vitality is the most significant asset.

Most vitality utilization is finished by information transmission related operations. Hence nearby handling of fundamental signs estimations and ceaselessly refreshing essential signs limits as indicated by the movement conveyed by the patient on every sensor hub, and sending information just when anomalous qualities exceed these reaches, can essentially decrease information transmission and spare the sensors' battery life.

\section{EXISTING SYSTEM}

The patient in ICU is observed by a specialist the information is been exchanged to pc is associated with wires .Hence this framework is troublesome. The framework are tremendous in size to such an extent that with the patient in the wake of releasing from healing centers and this framework can't be utilized by the individual .The information is not exchanged persistently for the quality nursing of the basically sick patient .Generally the essential parameters, for example, ECG, pulse ought to be checked by a specific individual and that ought to be noted in the patient record physically.

\section{Detriments:}

The estimations are taken physically, there is more odds of blunder happening. The constant qualities might be noted rely on upon individual and the onlooker .The upkeep of the database of the patient is dangerous undertaking.

\section{PROPOSED SYSTEM}

In this proposed framework we foundation a temperature sensor, Electrocardiogram sensor, glucose sensor and drowse sensor to get the subtle elements of the information when it cross the edge esteem. This esteem is shown on the LCD screen. On the off chance that patient is feeling any irregularity the automated insinuation will go to specialist .The implication SMS will be sent through the GSM module.

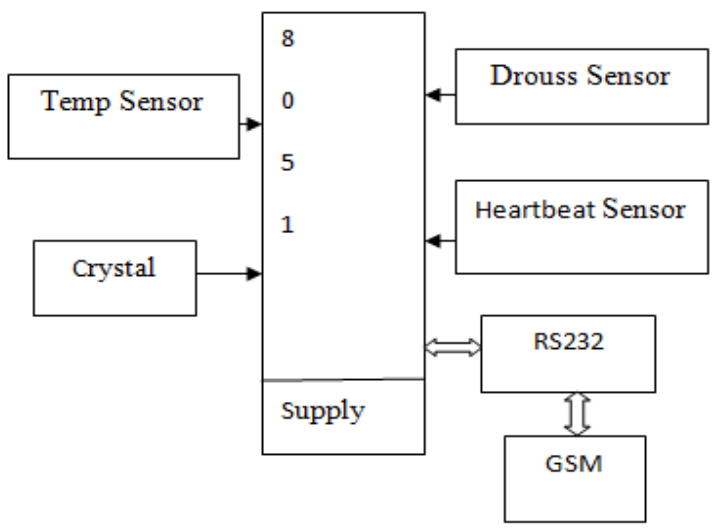

Fig 1: Transmitter architecture

\section{Hindrance:}

This can be executed by the home care persistent. It decreases the cost and builds the quality existence of patient .In technique no compelling reason to keep up the record of the patient physically.

Transmitter: Exchanging information to a remote place utilizing GSM and arm11 empowered cell phone.

Receiver: The collector end is outfitted with arm 11 empowered cell phone. Which is joined to the PC to check the status of the patient.

LM35 Temperature sensor: A temperature sensor is a contraption, regularly, a thermocouple or RTD, that obliges temperature estimation through an electrical banner.

Heart beat sensor: The basic heartbeat sensor includes a light releasing diode and a marker like a light perceiving resistor or a photodiode. The heart beat pulsates makes an assortment in the flood of blood differing regions of the body. BPM (Beats each minute $)=60 * \mathrm{f}$.

$\boldsymbol{E C G}$ : Electrocardiography (ECG) is the way toward recording the electrical movement of the heart over a timeframe utilizing terminals set on the skin. These terminals recognize the small electrical changes on the skin that emerge from the heart muscle's electro physiologic example of depolarizing and depolarizing amid every pulse. It is a usually performed cardiology test.

Microcontroller: Microcontroller is the mind of the venture, it will keep running as per the program 
must be composed, here we have utilized as a 89C51RD2BN Microcontroller.

Display: we are utilizing 16x2 LCD show, it will show the Latitude and longitude.

MAX 232 IC: This unit has MAX 232 IC, which is used to pass on between microcontroller unit and the GSM modem.

It trades the data to GSM and from the $\mu$-controller unit. This unit changes over microcontrollers TTL signals into RS232 hail sort as the GSM modem needs RS232 signals.

GSM modules: GSM, which remains for Global System for Mobile correspondences, rules as the world's most broadly utilized phone innovation.

\section{Implementation}

In our project we use an four module they are as follows:

- Sensor Manager Module

- Threshold Manager Module

- Contact Manager Module

- SMS Manager Module

\section{Sensor Manager Module}

A sensor is a gadget that measures a physical amount and changes over it into a flag which can be perused by an eyewitness or by an instrument. In this venture we utilize accelerometer which is implanted in PDAs.

\section{Threshold Manager}

- Threshold Manager makes it easy to set up events and cautions (limits).

- A edge is a trial of some element against some regard, with a report when the farthest point regard is outperformed. So it scales to the greatest frameworks, with little development overhead.

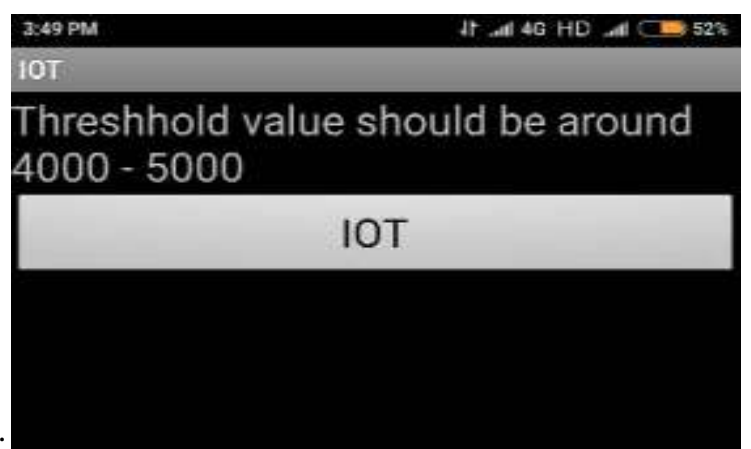

Fig 2: Threshold manager

\section{Contact Manager Module}

- The module reveals how to add contact to the social contact list. The customer is given the option of including more than one social contact to the summary.
- When the emergency is recognized the message is sent to each one of the connects with one by one. There is more over the decisions given to the customer so that the customer may eradicate the contacts and besides the incorporate contacts.

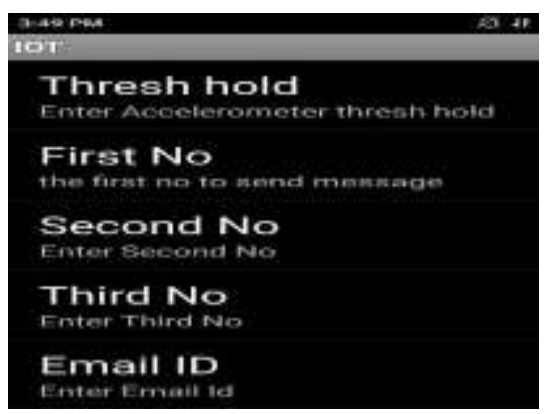

Fig 3: Contact manager

\section{SMS Manager Module}

- Text illuminating, or informing, permits to the exchanging of brief made messages between settled line phone or wireless and settled or minimal contraptions over a framework.

Applications

- It can be worked remotely by interfacing a modem framework.

- It can be used as a piece of ICU's, operation theater checking of oxygen levels etcetera.

- It can be additionally utilized as a part of seniority homes to screen the different parameters of a wiped out individual in maturity homes.

- All the Parameters can be seen on the Mobile telephone

- 8 autonomous simple channels accessible, for future Enhancements.

- On-line illustrations for selectable parameters On-line recording of all procedure parameters in the interim selectable by the client, which is most helpful for future investigation and disappointment location.

- State-of-the craftsmanship innovation framework

- Supports countless sensors to the framework

A framework includes an arrangement of performing artists, UML has a graphical documentation for condensing use cases. As the above figure appears, client have the entrance to get a message and know the patient status and status and different process are procedures are performed by controller which will assume a vital part in the whole execution. 


\section{FLOW CHART}

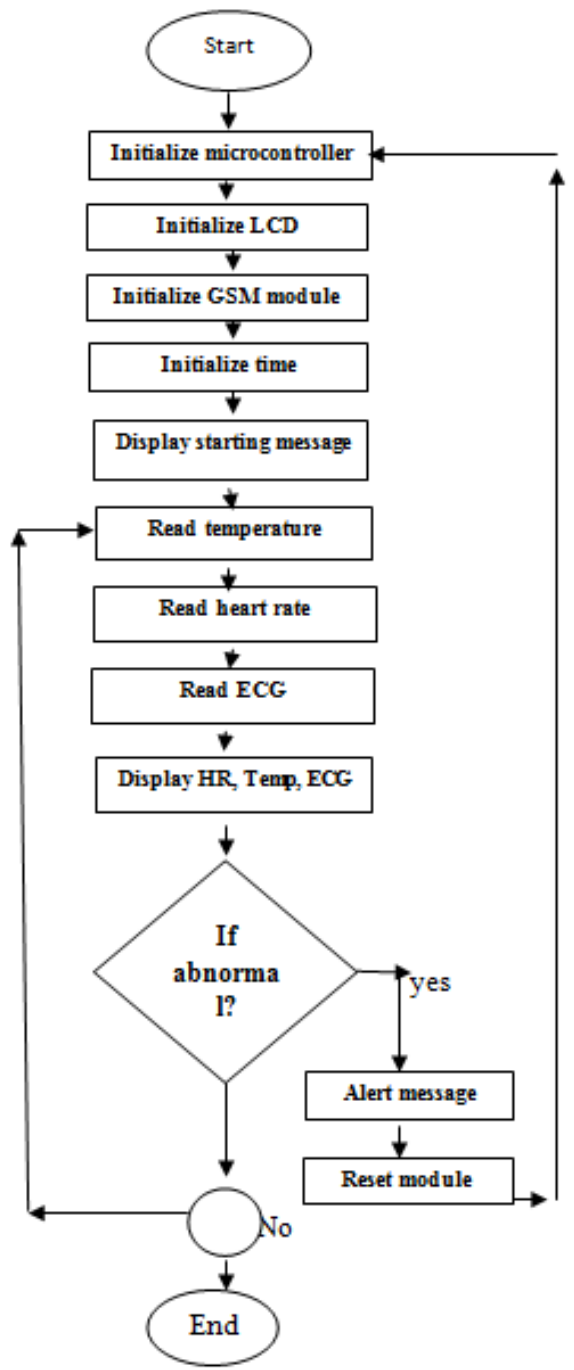

Fig 5: Flow chart for patient monitoring system

\section{CONCLUSION}

In this proposed model of watching physiological parameters, for instance, temperature, beat, ECG, glucose, are more viable than right now open structure. Starting at now available systems for watching physiological signs encounter the evil impacts of specific imperatives. The proposed structure is an immense change over existing business techniques, the present system can support up to twenty patients with continuous, low-control, negligible exertion, long-evacuate, and twofold mode checking, from the above made wander. The keil $\mathrm{C}$ writing computer programs is utilized for executing the method and results were inspected. In future we can amplify this structure by utilizing RFID development; through this advancement we can screen the different amounts of patients. It may be a future work to develop another patient checking application code toward that way.

\section{REFERENCES}

[1]. Karandeep Malhi, Subhas Chandra Mukhopadhyay, Fellow, IEEE, Julia Schnepper, Mathias Haefke, and Hartmut Ewald "A Zigbee-Based Wearable Physiological Parameters Monitoring System" IEEE sensors journal, vol. 12, no. 3, march 2012 online at http://ieeexplore.ieee.org

[2]. b. sirisha, t.sraddha, k. vijayanand "Realtime multi-patient monitoring system using arm and wireless sensor network" International Journal of Communication Network Security, ISSN: 2231 - 1882, Volume-2, Issue-2, 2013

[3]. Reza S. Dilmaghani, Hossein Bobarshad, M Ghavami, Sabrieh Choobkar, and Charles Wolfe "Wireless Sensor Networks for Monitoring Physiological Signals of Multiple Patients" IEEE transactions on biomedical circuits and systems, vol. 5, no. 4, august 2011 online at http://ieeexplore.ieee.org.

[4]. Yadav Satyendra Satyanarayan, Yadav Raghvendra Satyanarayan, Deep H. Desai "Intelligent Wireless Emergency Alert System for Patient Monitoring using AT89S52 Microcontroller" International Journal of Advanced Research in Electrical, Electronics and Instrumentation Engineering Vol. 2, Issue 4, April 2013 online at www.ijareeie.com

[5]. Juney M George1, Venugopal G "Design of an embedded platform for patient monitoring applications using $\mu \mathrm{c} / \mathrm{os}$-ii” International Journal of Advanced Research in Electrical, Electronics and Instrumentation Engineering Vol. 2, Special Issue 1, December 2013 online at www.ijareeie.com 